\title{
Interacting with Info-graphics in a Print-based Environment
}

\author{
Claire Timpany \\ Computer Science Department \\ University of Waikato \\ Hamilton, New Zealand \\ ctimpany@waikato.ac.nz
}

\author{
Nicholas Vanderschantz \\ Computer Science Department \\ University of Waikato \\ Hamilton, New Zealand \\ vtwoz@waikato.ac.nz
}

\author{
Ben Hallahan \\ Computer Science Department \\ University of Waikato \\ Hamilton, New Zealand
}

\begin{abstract}
Technology and our experiences with it are constantly evolving, therefore our views of how media can enhance our experiences with the information it presents to us must also evolve. Designers are able to learn from these "new" digital experiences in order to reinterpret and apply new methods and theories to traditional print based information technologies for the benefit of the user. This research endeavours to develop an enhanced method of presenting and navigating statistical information in a printed medium through the use of interactive methods. The fields of print, hypertext and information visualisation inform the development, with a view to create cognitive awareness in readers, as well as their ability to build information relationships. In both hypertext environments and information visualisations the clarity of context and structure play key roles in the successful presentation of the relationships between aspects of information. Through the process of a DesignAnalyse-Refine methodology, a range of designs for unifying these forms of media were explored and analysed. This produced a single design solution where the strengths of print are being interwoven with those of hypertext and data visualisation.
\end{abstract}

\section{Author Keywords}

information visualisation, hypertext, interaction in print

\section{ACM Classification Keywords}

H5.2. Information interfaces and presentation (e.g., HCI): Miscellaneous.

\section{INTRODUCTION}

In today's information rich age new techniques are employed to present often complex information to the ever more information savvy consumer. The advent of information technology has enabled rich digital information viewing experiences for the everyday user.

By crossing the fields of print, interaction design and information visualization, this research investigates the possibilities of employing interactive methods of hypertext to enhance the presentation and navigation of statistical data in a printed medium. Creating the potential to provide new innovations for viewing and presenting statistical information as well as enhanced content navigation, comparison, and knowledge connections. The importance of this is its ability to create non-linear learning opportunities, which can be unique to the reader. It also unifies the physical properties of the book with the principles of interactive systems. Purchase et al. (2008) views information visualisation as a means of communicating from a data set to the "cognitive processing centre" of the learner's mind. Pluralism, a cognitive approach that teaches learners to view information from various perspectives, is also the focus of hypertext where perspectives and points of view can be shown in the arrangement of nodes (Beeman et al., 1987) As found in this research, the combination of these concepts is ideal for enhancing the reader's cognitive and learning capabilities, while improving users ability to build information relationships.

\section{Information Visualisation}

Information visualisation is best described as a "representation of abstract, non-physically based data to amplify cognition" (Eden, 2005, p. 7). It is a process of transforming information into a visual form that can make use of man's natural ability to rapidly identify patterns (Gershon \& Eick, 1997). Visualising information makes presenting complex concepts possible; using a physical visual representation to do what visual and verbal memory cannot (Keller \& Tergan, 2005).

Statistical information is often complex, meaning viewers can struggle to make meaningful connections with the information presented to them, and develop a clear understanding of that information. The transference of knowledge in information visualisation is based upon four principles: concept, structure, context, and navigation Woolman (2002).

Perhaps the most important principle of visualisation is the decision regarding how to structure the information on the page. The structure of an information visualisation is vital for conveying the inherent relationships of the data (Tufte, Goeler, \& Benson, 1990). Context provides the place in which the structure of the information takes shape. The representation of the context can be either literal, like the locations on a map, or metaphorical, like the bonds between different ideas (Woolman, 2002).

The benefits of information visualisation are many and varied and Fekete et al (2008) points out the core benefit of visuals seem to rely upon them behaving as a temporary storage area in the mind of the learner to allow them to process the information. This explains that the 
use of a visual construct means that the learner has a single focal point to draw from, not a drawn out sequence of text. Klanten et al. (2008) state that the use of visuals acts as an analogy, utilising what is simple and familiar to aid in the comprehension of what is complex or unknown. They claim that any composition of information "must ensure comprehension" (p. 8) otherwise the meaning of the complex relationship is lost. It mirrors hypertext in some respect as it provides a great deal of information at once that can be navigated at will to draw conclusions relative to the learner's needs with all the benefits of print.

\section{Interaction Design}

Print media and technology driven interactive environments each have unique strengths, yet it is their common attributes that act as motivation for this research. There is nothing that dictates that interactivity has to be technology driven (Cover, 2006). Translating interactive methods into print has the potential to be effective, but needs to be carefully structured to function effectively as was seen in Timpany (2012). Here Timpany explored the relationship between hypertext and the index and investigated suitable methods of translating the concepts of hypertext to print to benefit learners.

Interactivity can be defined in many different ways (Downes \& McMillan, 2000; Kiousis, 2002; Kirsh, 1997). The definitions used in the literature depend on the context, the purpose of the definition and the medium or technology that is being discussed. "Physical interaction corresponds to things which achieve related changes in state in the physical world" and "Communicative interaction concerns things which could achieve changes of state in the mental world" (Elsom-Cook, 2001, p. 9). The principles of context and structure are important in creating interactive environments where users can efficiently achieve their intended goals, whether these are physical or communicative interactions.

A good interface design, whether printed or digital, should take the user on a journey where they are gently encouraged to experience the interface and it's content. "The goal of an interactive interface is not merely to allow users to do what they want to do; it must allow them to discover what they want to do" (Kirsh, 1997, para. 22). For interactive information visualisations, effective execution of an interface that encourages experiential interactions does not ensure success, as the presentation of information is equally as significant.

\section{Hypertext and Information Visualisation Relationships}

Hypertext is an interactive system whereby a user can navigate between units of information which have relevance to each other. This is achieved through connections, creating links between words or passages in text. Hypertext structures in text enable the same piece of information to be represented in multiple contexts. Context is an important aspect of comprehension for interpretation of meaning (Bieber, 2003). The data in a hypertext system gains meaning and context through the relationships it has with other data (Landow, 1997; Miles, 2001). Creating clear navigation is an important consideration for the development of successful hypertext systems.

Focusing and linking, at their simplest, "are principles that offer a solution to the problem of visual overload" (Buja, McDonald, Michalak, \& Stuetzle, 1991, p. 161). This means that the information can be spread into manageable, understandable segments, which relate to a single aspect of the overall data. Spreading the content out in this manner introduces another very important method of information visualization: utilising multiple views.

Utilising multiple views of the same content can make identifying the varying layers of content a subconscious process, not a laborious one. Chalmers (1995) adds "by making the transitions between levels smooth and continuous we retain context as we zoom in on some detail, and therefore maintain orientation" (p. 6). Then as the user moves from view to view the overall construction is still comprehensive, yet still fluid enough to allow the user to build an image relevant to them.

The multi-level structure of a hypertext document allows for text acquisition, storage, analysis, comparison, retrieval and editing by the user. Hypertext environments actively engage the reader in the creation of meaning and structure in a text. Through the relationships expressed in a hypertext document greater context is able to be created for the reader.

A close resemblance to hypertext is evident in interactive information visualisation; it also provides the user with the ability to retrieve information through the embedded links (Kraidy, 2002). In the design of interactive media the experience of the user is paramount. Concept, structure, context, and navigation, according to Woolman (2002) are also important to information visualisation, and become significant in enhancing user epxerience. The information visualisation principles of structure, context and navigation correlate and overlap with the principles of hypertext, indicating that the presentation of visualisations in the static medium of print have the potential to be viewed in a dynamic manner.

\section{METHODOLOGY}

To explore the potential of creating interactive infographics in a print-based environment a Design-AnalyseRefine methodology, as used by Vanderschantz (2008), was implemented. Of central concern to this study was to investigate a range of novel solutions and refine the effectiveness of the interactions.

The Design-Analyse-Refine (DAR) methodology is a practice-based methodology that allows for a cyclic sequence of development through a number of rounds. A round is a specific phase of the methodology, including design, analysis, and refinement phases, before new designs are created for the next round, as demonstrated in figure 1. Both the design and refinement phases were carried out by the researcher; the analysis was carried out through critique by three experts. These experts were each skilled in their own fields of graphic design, 
hypertext structure, and data visualisation, mirroring the three focal points of the research.

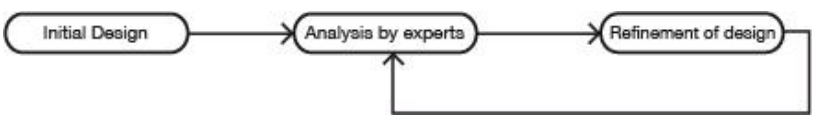

Figure 1. Stages of the Design-Analyse-Refine Process

The experts were asked to interact with each design and then provide feedback pertaining to its usability, effectiveness, and areas for further improvement. The sequence of interactions and responses was tailored to attain their preconceptions, reactions during use, and responses to the system as a whole. From the experts' analysis of these designs the most advantageous aspects of each design were developed for the next round, taking into consideration potential improvement and refinement.

There were four rounds of design, analysis and refinement, the first round begun with four designs as potential solutions to the proposed problem, by the fourth round this had been refined to three. In the final round one design was unanimously chosen as the strongest, the experts agreed that its levels of structure, connectivity and content were matched by its freedom and openness to the user's needs. The progression of designs can be seen in figure 2 .

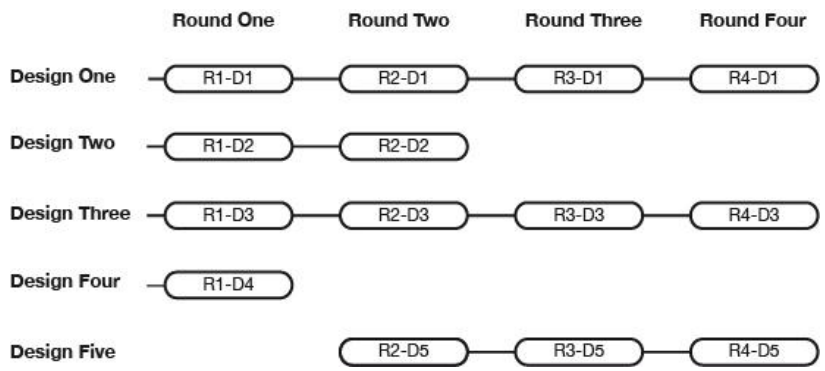

Figure 2. Progression of Each Design from Round One

The data chosen for use in this experiment was statistics from the qualification and final tournament of the 2010 FIFA World Cup, as it provided a consistent set of statistics as well as a fixed range of search parameters.

\section{EXPERIMENT}

\section{Round One}

For the initial round of design-analyse-refine the aim was to investigate four different methods of information visualisation in four different formats. Another consideration was the navigation method to be incorporated into each, with a view to find one that not only functions well for the design, but effectively aids movement throughout the content.

\section{Design One (R1-D1)}

This design was developed specifically to give the user the freedom to navigate individual team appearances. The visualisation method was based primarily on typography, and the format used was a ring-bound unit of two sets of cards that were flipped over or removed for comparing content. The navigation method was an alphabetical tab system. This meant the user interacted by aligning the cards side-by-side to compare a team's performances, or contrast with the opposing teams.

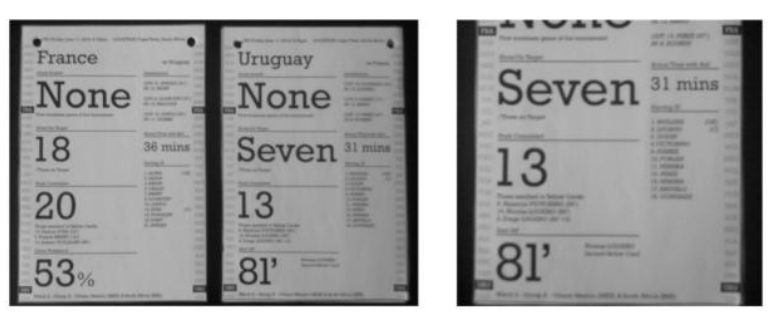

Figure 3. Round One - Design One (R1-D1)

The first design was deemed easy to use by the experts. The majority of feedback discussed the layout more than the navigation or comprehension. Expert One suggested that the cards be modified to contain the content of multiple games and could, perhaps, be rotated to align with other teams. Experts Two and Three felt that the cards were too small, suggesting that providing more space for content and hierarchy would aid in navigating the information and improve access to the content. The cards functioned like nodes, but the linking aspect was still somewhat lacking.

\section{Design Two (R1-D2)}

Design Two consisted of three books, separating the tournament into three logical sections, containing groups A-D, groups E-H, and the knockout stages. The tab-based navigation was separated into groups, the groups A-D used the top half of the page, and groups E-H used the bottom half. Separating the tabs gave an alternative method of grouping that was relevant to the content. Aiding this was the colour coordination of the three books, so they could be easily distinguished. The visualisation method used was 'geometric' and used a central visualisation to present 'possession', 'shots' and player infringements in a single visual.
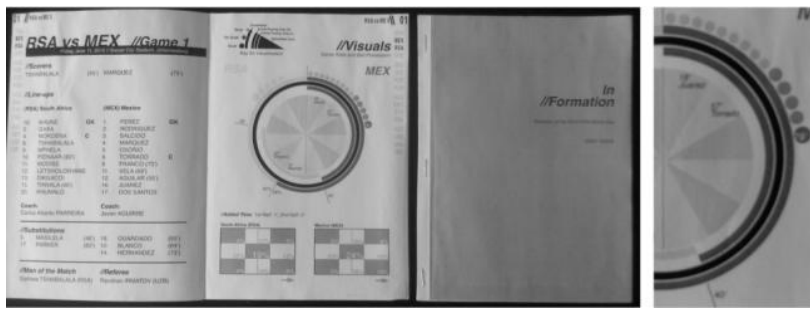

Figure 4. Round One - Design Two (R1-D2)

Design Two had mixed reviews, with the format criticized for its restriction on the content, much like R1D1. The navigation was also thought to be difficult and the separation of the books was not as effective as it could be; suggestions were to break them into rounds. While the format and navigation had issues, the visualisations had great potential and could be refined to present multiple dimensions easily. Expert One felt the visualisation, presenting multiple dimensions of match information, was very effective. 


\section{Design Three (R1-D3)}

The third design of Round One, aimed toward the football aficionado, was a larger, spiral bound book that conveyed in-depth data. The visualisation method combined both spatial and temporal elements, with the match shown relatively in a linear timeline. This was aided by a timeline for the entire tournament, showing the current progress of the team. Including a 'goal map' instantly giving the user context for the action. Navigation in this design was user-defined, with perforated tabs that could be marked out to identify content the user deemed significant.

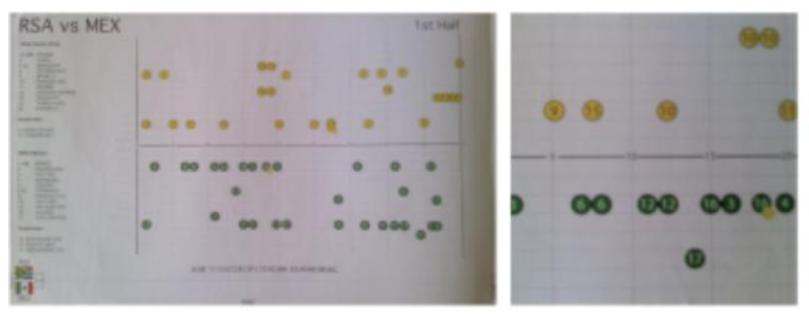

Figure 5. Round One - Design Three (R1-D3)

Feedback on the third design was positive, but the experts commented on the lack of detailed statistical content. Experts One and Two agreed the goal map and timeline were effective in conveying spatial and temporal data, but the navigation system was less effective than in previous designs. The visualisation itself was more in-depth, and this complexity and detail was believed to have potential. While the presentation of time-based content was welcomed, Expert One commented that it lacked any relationship to the surrounding content. The navigation was difficult to use effectively and while being customizable, it lacked structure, which could potentially confuse a new or less-prepared user.

\section{Design Four (R1-D4)}

The fourth design was square in shape with clear in-page navigation that was user-oriented. It used coloured adhesive tabs to mark particular categories of information. This was aided by large orientation elements on each spread.

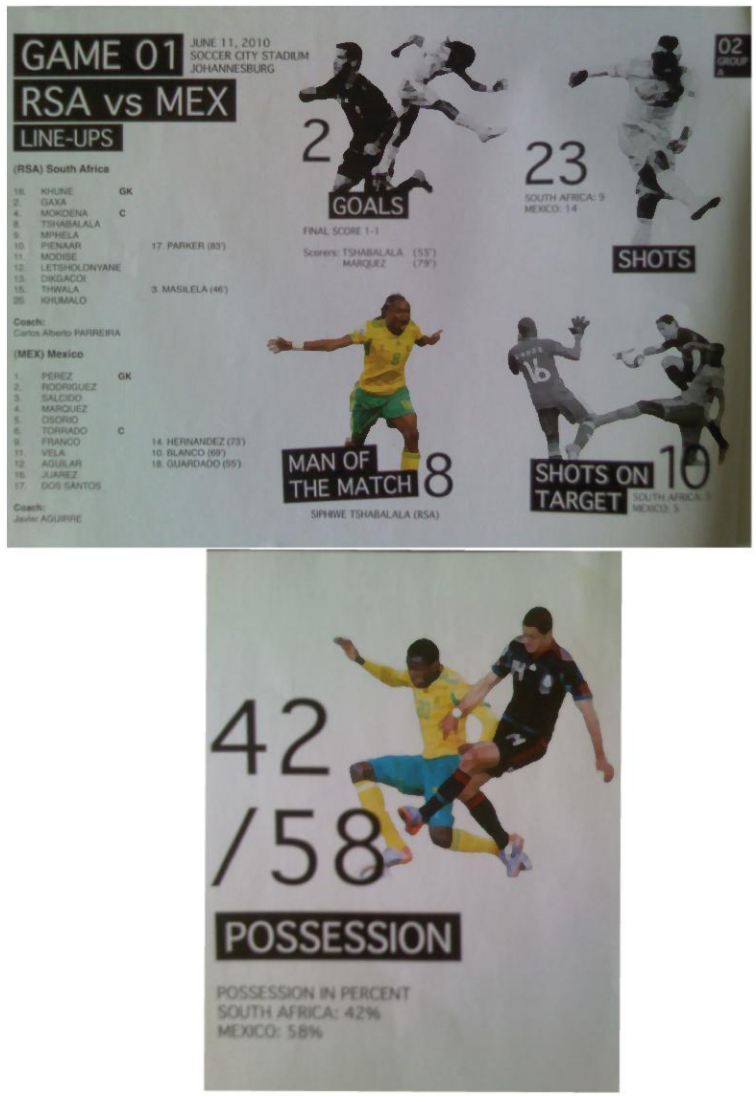

Figure 6. Round One - Design Four (R1-D4)

Design Four was believed to be more usable for the casual reader, as the content was simplified greatly. Expert Two found that the individual data elements lacked identifiable relationships, reducing the ability to compare and correlate. The user-developed navigation was questionable as it relied heavily on the users own concept of structuring related content. In contrast Expert Three felt this design had broader appeal, and combined with user-built navigation provided a more personalizable book. This design seemed to be the weakest, and while the navigation method was versatile, it would be more useful as a supplementary device. With this in mind this design was not retained in the process.

\section{Round Two}

When developing the experimental designs for the second Round suggestions from experts on each design from the first Round were taken into consideration. These included refining the content hierarchy for each design, focusing on navigation, and adding a player or team index and summaries to provide greater interactivity and structure. These comments by the experts emphasized the importance of structure, context and navigation as was also expressed by Woolman (2002).

Design One (R2-D1)

The first design of this Round came directly from R1-D1, but the layout and content were reduced from two cards to one. The single card now utilised both sides containing type-based information and a field layout on the reverse. It maintained the alphabetical navigation system, but was now aided by team colours in each side, meaning cards 
could be aligned vertically to show a team's progress. The binding system was modified, making the side navigation easier to use.
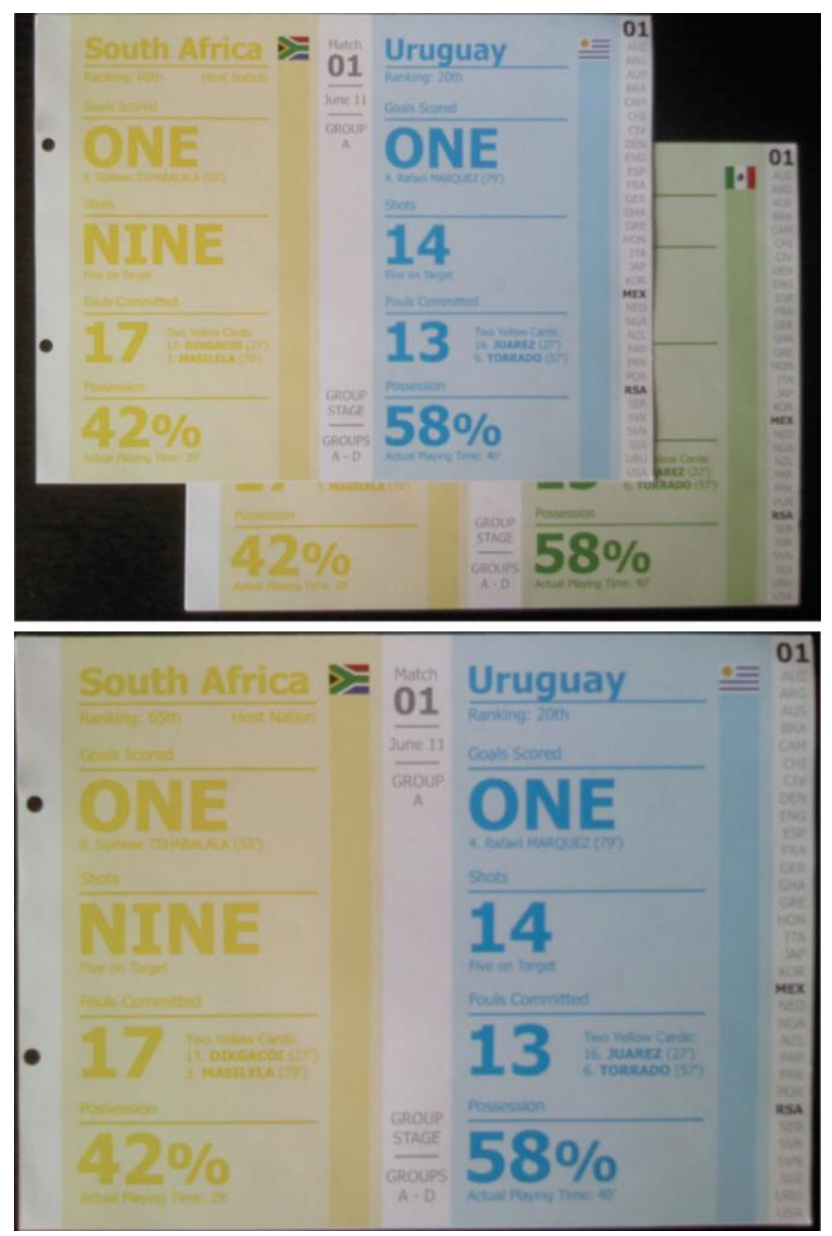

Figure 7. Round Two - Design One (R2-D1)

The experts gave the first design a more positive response in this round, and while it was not structurally complete it was better for comparison. The lack of sophistication in the aesthetic qualities was a concern for all three experts, as they felt this detracted from interacting with the information. Expert One felt that this design now had increased aspects of hypertext and hypermedia, which they considered very good for comparing the content from each team. However, Expert Two felt the connectivity could have been explored further with the two sections of information now presented on the same card, they failed to link to each other as well as to the corresponding team information on other cards. Expert One and Two both felt that comparison between individual elements needed to be easier.

\section{Design Two (R2-D2)}

This design translated directly from R1-D2, and was developed with layout in mind. The 'team line-up' was incorporated into a 'pitch map', to aid the understanding of the players and their positions. The layout itself was less cramped and there was greater importance placed upon visuals, with the focus placed on the main visualisation.
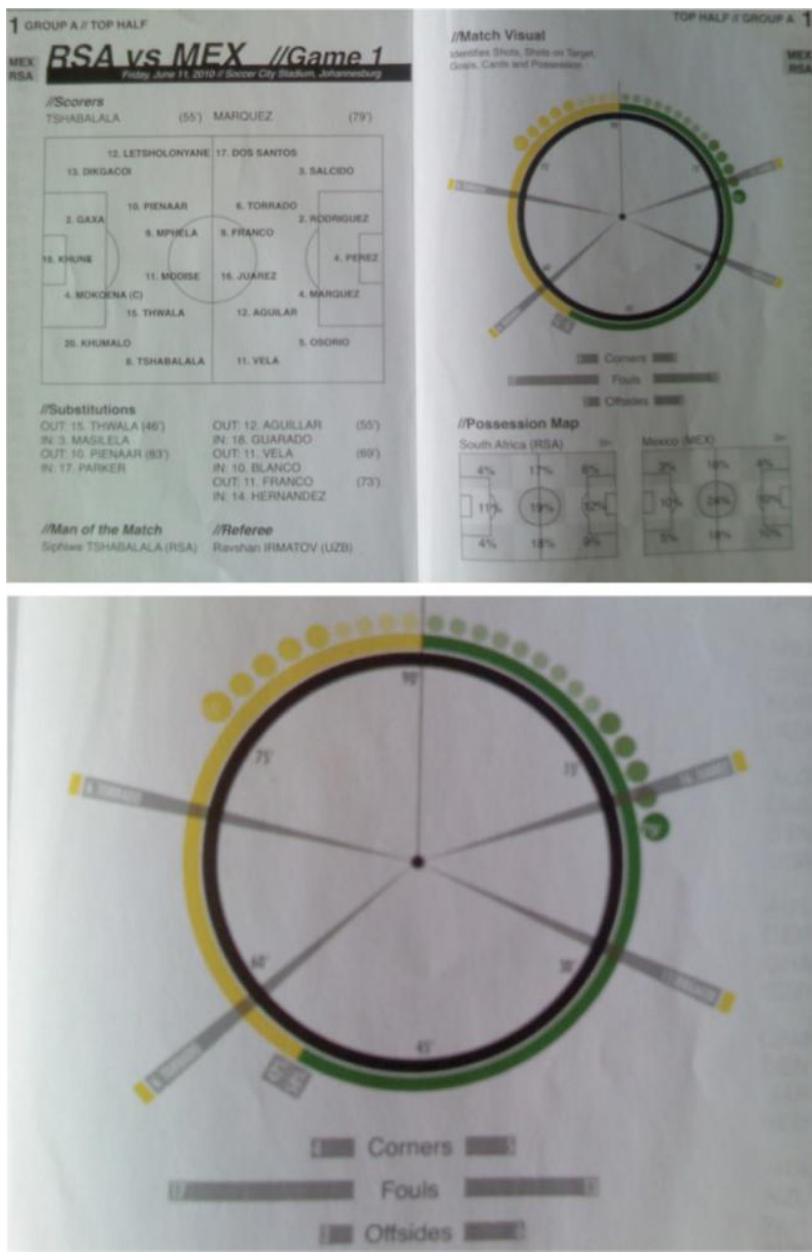

Figure 8. Round Two - Design Two (R2-D2)

A common impression from the experts about 2 was that the infographic was multipurpose and had the ability to be developed further to accommodate a range of data dimensions. Expert One was impressed by the multipurpose visualisation, he liked the variety of dimensions being presented and thought encoding the shots in the timeline could enhance this, giving it similar qualities to R1-D3. Despite the success of the visualisation, the format was considered unsuitable for further development so was discontinued, but successful elements were incorporated elsewhere.

\section{Design Three (R2-D3)}

Design Three developed significantly for this round, with a hierarchy more befitting the timeline's needs. The page statistics were laid out with greater care and the orientation was clearer and consistently located. The timeline of the tournament was relocated to show the overall context of the match being presented. For the navigation, the user-created system from R1-D4 was reintroduced. 

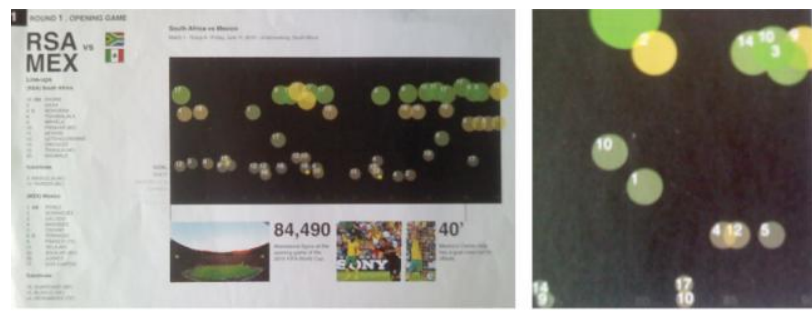

Figure 9. Round Two - Design Three (R2-D3)

Design Three was still considered to be consistent, with an ideal format maintained, the content and visualisations had been vastly improved according to the experts, suggestions for improvements were very clear though. Experts One and Two suggested linking the timeline goals to the map, building the relationships and showing the correlation between spatial and temporal information. They also suggested that there could be greater contrast between positive and negative play, using contrasting shapes to convey this. Expert Three felt that the timeline would be improved by adding a marker to help identify the current point of the tournament.

\section{Design Five (R2-D5)}

Replacing R1-D4, the pictorial design from the previous round, was a folding wall chart design. It had five tiers, from the group stage to the final, in a shape implying upward progression. The 'group stage' card contained a brief summary of the six matches within the group, as well as a 'points table' and key statistics. Linking was incorporated using coloured squares and paths to join related matches, as well as higher levels if the teams progressed.
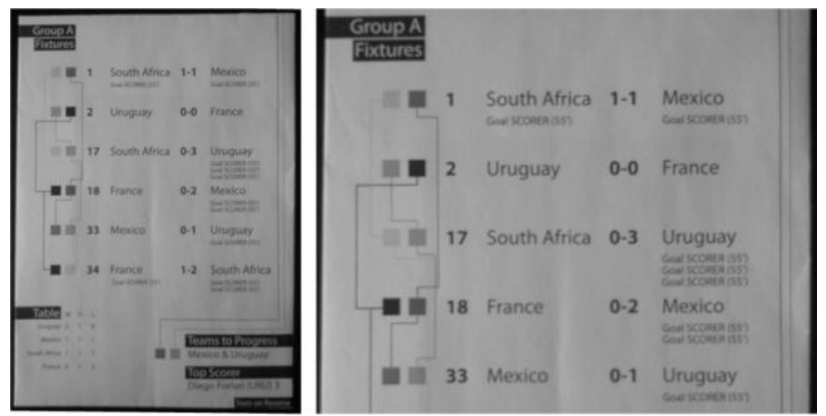

Figure 10. Round Two - Design Four (R2-D5)

Of the four designs this round, the newest had the greatest detachment from the standard book paradigm and received a lot of feedback regarding its layout and content. Linking of panels needed improvement, so incorporating flags and relocating them would aid this dramatically. Expert Three commented that using the flag as a match marker will also aid the identification of a team's appearance. The node-like layout helped to structure the wide range of information being presented. The experts felt that while it had great potential the logistics of an elaborate construction like this would have to be considered carefully.

\section{Round Three}

At this stage of the process, five different designs had been introduced and critiqued, Round Three begun to focus more closely on three specific designs, reducing from four down to three. At this point most of the navigation had been refined and the layouts determined, so the focus was on arranging the content and improving the flow of each design. The content and arrangement of the information is primarily focused on creating context and the structuring of the content, as Woolman (2002) described as important aspects of information visualization and often are closely linked..

\section{Design One (R3-D1)}

A card-based design was developed and absorbed elements from the discontinued designs from earlier rounds, such as the main visualisation from R2-D2 and imagery from R1-D4. The front face included the same information about two teams, which was now aided by the main visualisation from R2-D2. Team colours were more restrained than previously, allowing the content to take the fore. The reverse had three clear elements: 'team line-ups', 'possession map', and imagery.
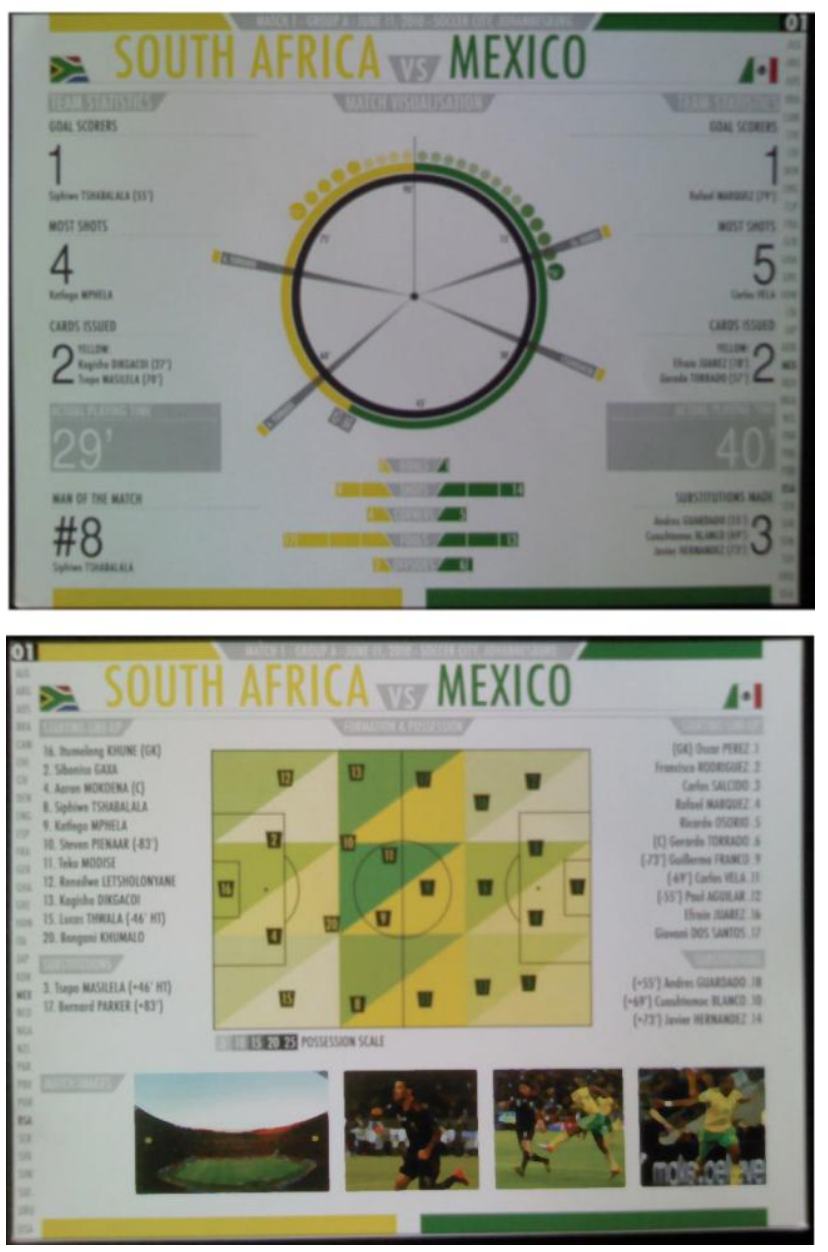

Figure 11. Round Three - Design One (R3-D1)

Many of the changes suggested in this round involved arranging the tournament structure and search function designs. It was suggested by Expert Two that adding a greater margin for the navigation would afford it a much greater chance of being utilised effectively. The hypertext 
aspect was considered to have developed very well and it was decided this would be tested fully in the next round. The aim was to refine the card layout and visualisations as much as possible.

\section{Design Three (R3-D3)}

Refinement was the key for this design in Round Three, and the typography was considered so that player names and numbers were easily related to the numbers in the visualisation. Also, the statistics were repositioned at the foot of the spread, to flow along with the timeline. Added to this design is the 'possession map' from D1, into the space below the 'goal map'.
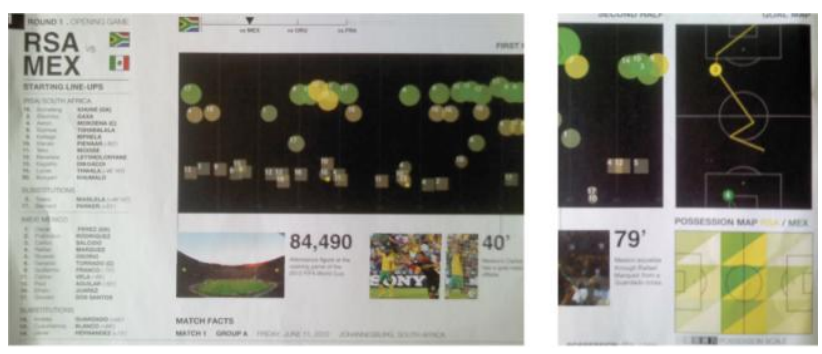

Figure 12. Round Three - Design Three (R3-D3)

For Design Two most of the recommended changes were aesthetic. Expert One discussed alternative possibilities for binding to make the double page spreads more interactive. Experts Two and Three suggested that all of the team's matches should appear on the timeline to show their full journey through the tournament, with those at later stages in grey. Placement was also a key aspect in the refinement of this design, as the horizontal flow guided the understanding of the visualisation. A design like this, tailored for the aficionado, needs to be able to be navigated easily.

\section{Design Five (R3-D5)}

Design Five presented the wall chart idea again, but placed greater emphasis on the modular layout and interconnectivity of the cards. The structure was reconsidered so that the progress of teams was more easily managed, and the summaries and statistics positioned in a logical flow. Alongside this, the national flags allowed for simple identification.
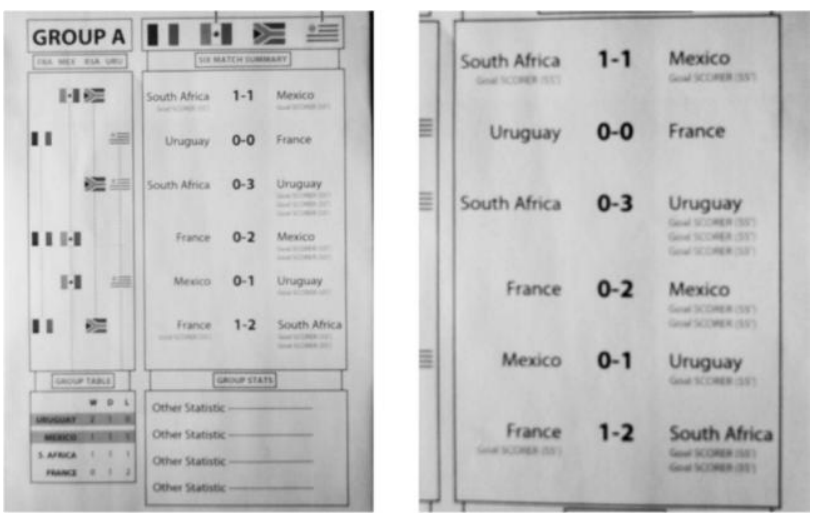

Figure 13. Round Three - Design Five (R3-D5)

Design Five was less refined than the previous two in the Round, but Expert Three felt that one of the key strengths of this design was the summary page. The key points made by the experts when reviewing this design revolved around the page layout and the linking between the levels of the tournament, which is important for providing context in hypertext. Expert Two felt it was important to maintain the module-type construction of each panel in the wall chart, mirroring the structure of hypertext. Expert Three believed that while the pages flowed well vertically, there needed to be better lateral connectivity.

\section{Round Four}

The final round of the design-analyse-refine process contained the three designs from the previous round; all with further development. With the navigation somewhat finalised, and the search function in development, the layouts and refinement were the focus of this round. The refinement of structure and context at this stage od the design process is most closely related to the metaphorical connections that Woolman (2002) describes which will assist the reader or viewer with creating meaningful connections between aspects of information within the layouts and between spreads.

\section{Design One (R4-D1)}

For the fourth incarnation of this design, consideration was given to the placement of the team statistics and visualisations. The individual team statistics were aligned to allow quick comparison. Enhancement was made to the central visualisation to aid comprehension of action in time, forming a circular version of R3-D3's timeline. The orbs around the edge were placed in time with their occurrence, meaning that the user can identify trends and periods of activity. The navigation was also refined.

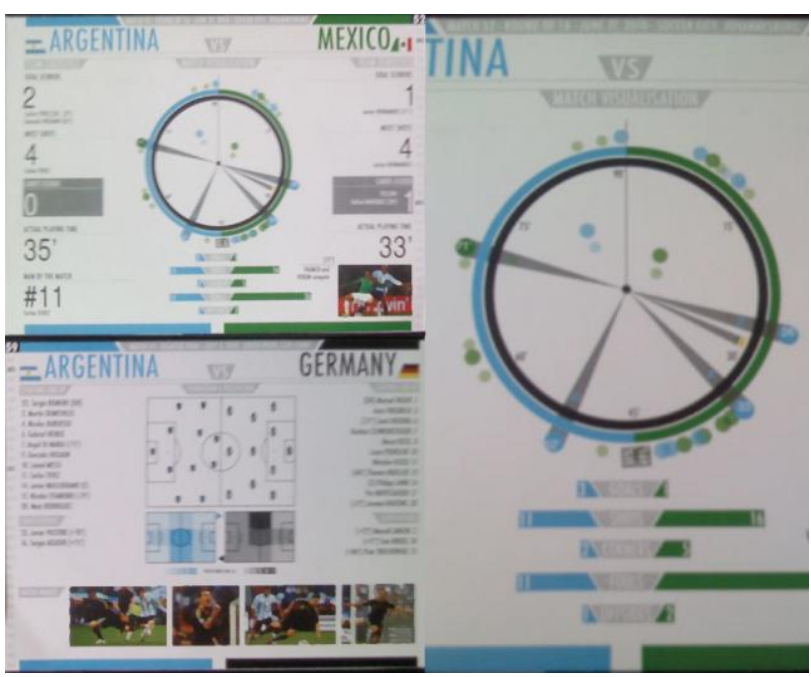

Figure 14. Round Four - Design One (R4-D1)

Design One contained a great deal of information and was the amalgamation of R1-D4 and R2-D2. The layout allowed for horizontal and vertical movement, meaning individual exploration was unrestricted. The central visualisation in this design was deemed to be the strongest and most refined of the round. Perhaps the most effective attribute of this design was the navigation and interlinking of content. Experts One and Two agreed that the ability to compare was strongest in this design. Expert 
One suggested that to improve this further the matching information should be aligned in a manner that allows for better understanding and speedier recognition.

\section{Design Three (R4-D3)}

For this round the modifications to Design Three focused on the visualisations. The timeline was lightened, meaning that significant acts in the timeline were more readily identifiable, and the links with the associated imagery were stronger, the R3-D1 'possession map' was also included. Hierarchy and natural progression were clearly present in this design, allowing the user to see content from before, during, and after a match in a logical sequence.

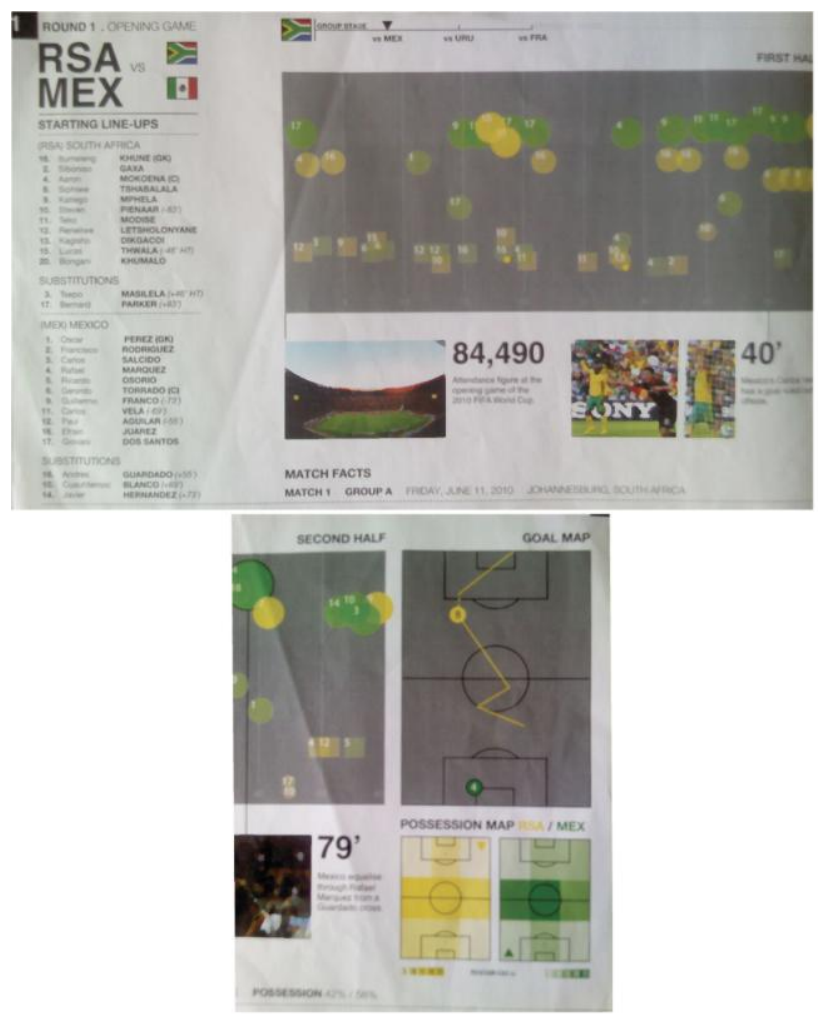

Figure 15. Round Four - Design Three (R4-D3)

Design Three was tailored more toward prolonged examination than glancing. All three experts noted its level of detail, but commented that it may also be a hindrance for less informed members of the target audience. The level of hypertextuality was questioned though, as the user-driven navigation left too much for the user to do. All experts were satisfied with the layout and content flow, suggesting only minor modifications to aid the ability to search. Format and visualisation complement each other well in this solution, but may be overly elaborate for a general audience.

\section{Design Five (R4-D5)}

Design Five was the most radically improved in this round, now showing the complete hierarchy of the tournament. Tournament groups were summarised clearly and concisely in a single panel, giving greater weighting to the later stages. The appropriate national flag accompanied each match, giving a symbolic image to aid identification. Below this was a small group table, providing an overview of game events. The advancing team flags were linked via paths to the round above, providing navigation between the tiers. The latter rounds were covered in more detail, with match statistics and more elaborate summaries included. The booklet unfolded into a pyramid shape, ideal for mounting on a wall and for understanding the tapering of the tournament.

The fifth design received positive feedback, but was felt to be still unfinished, and would rely too heavily on elements from the other designs. It impressed in its appropriate level of detail for each round, and Expert Three felt the group summaries were excellent and worth retaining in any of the designs. Unfortunately the layout was not to the level that was hoped for, and the structure was "contrived" and meant users were bound by the ready-made construction. Although it was less refined than the previous designs, it gave a great deal of insight into what further progress could be made in the final solution.

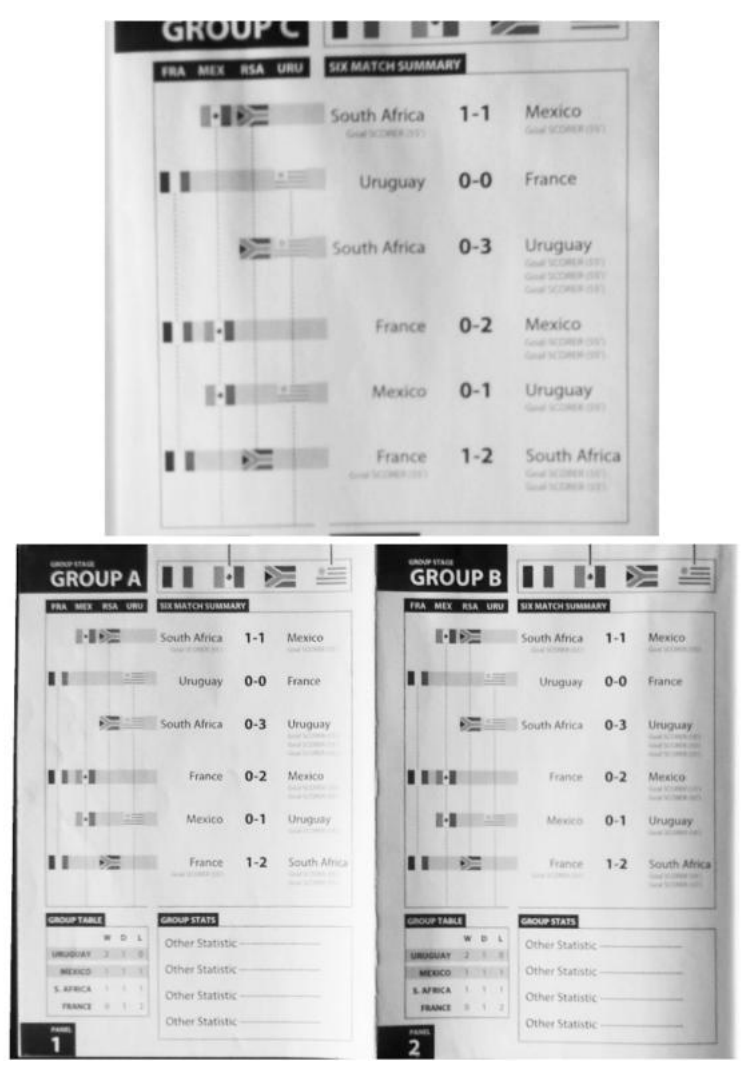

Figure 16. Round Four - Design Five (R4-D5)

\section{SOLUTION}

At the conclusion of the design-analyse-refine process, with assistance from the experts, the strongest design was identified to be refined into the final solution. The final three designs provided diversity and an understanding of the range of ways interactive info-graphics could be created in print. Design One was unanimously chosen as the strongest, the experts agreed that its levels of connectivity and content were matched by its freedom and openness to the needs of the user. Suggested refinements were incorporated into the solution as well as 
enhancements drawn from successful aspects of discontinued designs, and an auxiliary search function incorporated.

The final solution was a collection of cards, whose order could be rearranged at the user's discretion. The cards are identifiable by a unique card number and tab system regardless of their place in their bound form. Additionally, the cards can be removed and compared, allowing for added dimensionality. Navigational tabs along the foredge are aided by colour and numeric association to each team and player, linking to a unique summary card for each team. Additionally, a small number of adhesive tags are included, creating the classic bookmark that hypertext uses so well, meaning the user can find their favourite cards instantly.

The wall chart idea was maintained by adding numbered markers at the head and foot of each card which, when joined together, present the structure of the tournament. Each card has different markers meaning they line up in a single formation. The supplementary timeline from the second design, was incorporated at the foot of each card's reverse side to provide context. Each match has a marker to identify where it is in the course of the team's progress, along with dates and other matches. Also included from the second design, was the 'man of the match', 'positive player', and 'negative player' markers, which identify the significant players in both teams.

The most significant addition to the solution is the search function, exhibited in the standard game page by the horizontal marker by each player's name, as well as the current match marker. Each team has a summarising card, and a range of symbols identify players' contributions. This card functions as an index of the users favourite team and to assist with locating specific information using the match and player markers.

The principles of information visualization defined by Woolman (2002) and supported by the ideas inherent in the design of successful hypertext systems (Bieber, 2003; Landow, 1997; Miles, 2001) are evident through the design solution. The content has been considered, context has been developed for the information presented, structure has been developed and effective navigation methods have been a key consideration throughout the design process. The use of hypertext ideals assisted with the development of meaning and context for the information presented through the visual relationships created between the different aspects of the content and the structure that has been created.

The design-analyse-refine process has been successful in creating a suitable solution through extensive research and a wealth of expert assistance, meaning a strong solution has been reached that meets the criteria outlined at the start of this research.

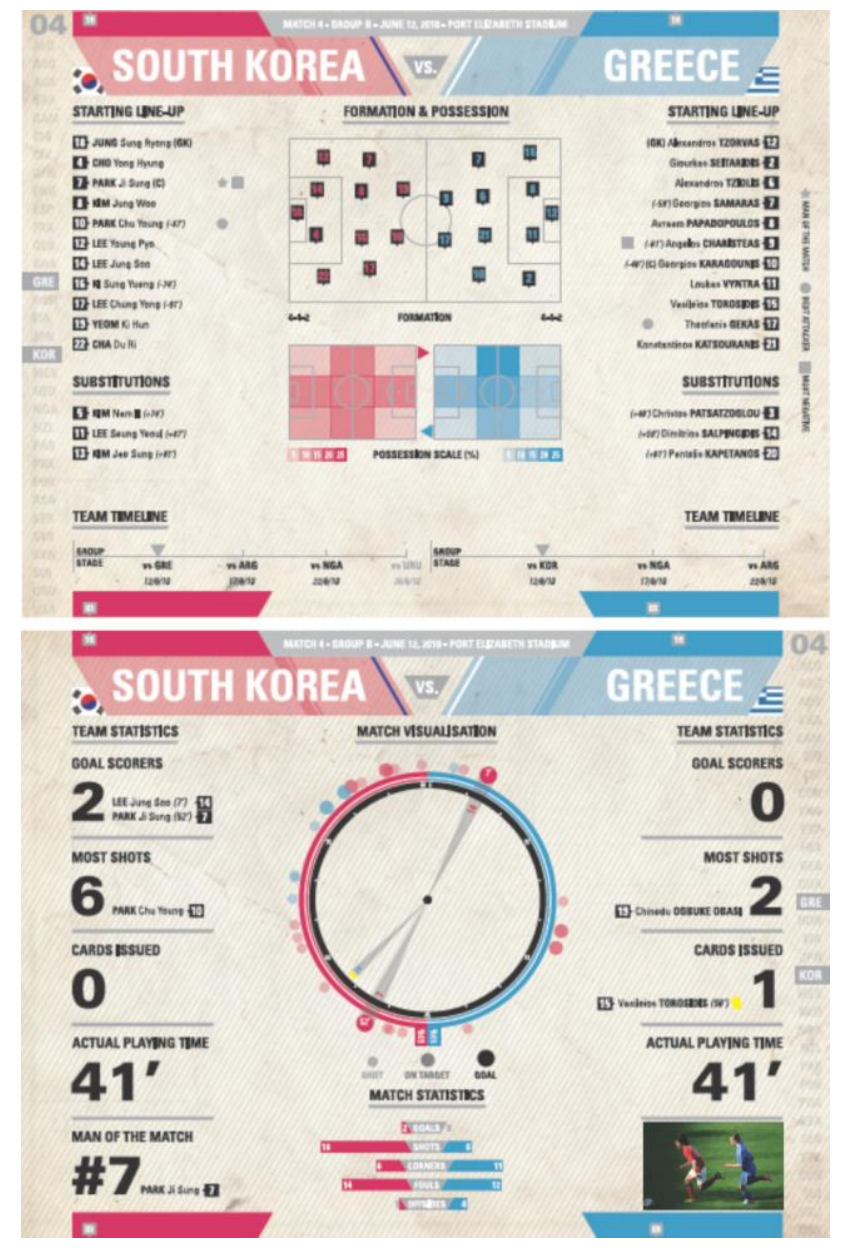

Figure 17. Final Solution - Front and Reverse

\section{CONCLUSION}

It is evident from the research undertaken that incorporating the principles and methods of hypertext does enhance the presentation and navigation of statistical information in a printed medium. The aim throughout was to unify the strengths of three unique media to create a visual and cognitive aid for the presentation of information. While the media are unique to themselves there are significant aspects of their structure that overlap, acting as the foundations of a potential solution.

Investigating the elements of hypertext and its potential as a navigation system in print revealed much about its use of structure and ability to assist a reader's comprehension of relationships and how context is constructed through links. Combining the strengths of print and hypertext create benefits which include conveying structure, aiding navigation, strong linking and the ability to search through the contents with ease.

Information visualisation plays a key role in the comprehension of complex knowledge in the modern world. In print, information visualisation is used to communicate and document complex concepts with a view to elaborate on specific aspects necessary to the viewers needs. By creating a visualisation of a confusing design, a learner is able to use it as a temporary storage area, allowing them to isolate certain areas to aid their 
comprehension, while still within the context of the overall composition.

One aspect that does not translate effectively is the ability to interact with the information freely, while the user can turn the page to the next visualisation, look closer, or label areas of interest they still have a somewhat detached relationship with the page. Remedying this requires the incorporation of hypertext, in this case harnessing its strengths and similarities with information visualisation.

At the conclusion of the design-analyse-refine process it was evident that incorporating the principles and methods of hypertext enhanced the presentation and navigation of statistical information in a printed medium. This reinvigorates print while utilising interactive concepts to aid the reader in a print-based environment.

Much like the work of Timpany (2012) this work showed that there are many potential ways that digital principles can be applied in print based media. This work extended these ideas further and explored how the use of the information visualisation principles of concept, structure, context and navigation, as described by Woolman (2002), can also be applied effectively in interactive print applications. Future work is required to understand more fully the multitude of methods for applying information visualisations using interactive principles in a printed form and the visual expressions of these ideas. This can be explored by way of further DAR investigations, user testing and field implementations.

\section{REFERENCES}

Beeman, W. O., Anderson, K. T., Bader, G., Larkin, J., McClard, A. P., McQuillan, P., \& Shields, M. (1987). Hypertext and pluralism: from lineal to non-lineal thinking. Proceedings of the ACM conference on Hypertext, HYPERTEXT '87 (pp. 67-88). New York, NY, USA: ACM. doi:10.1145/317426.317434

Bieber, M. (2003). Hypertext. Encyclopedia of Computer Science (pp. 799-805). Chichester, UK: John Wiley and Sons Ltd. Retrieved from http://dl.acm.org.ezproxy.waikato.ac.nz/citation.cfm?id $=1074100.1074458$

Buja, A., McDonald, J. A., Michalak, J., \& Stuetzle, W. (1991). Interactive data visualization using focusing and linking. Visualization, 1991. Visualization'91, Proceedings., IEEE Conference on (pp. 156-163). Retrieved from http://ieeexplore.ieee.org/xpls/abs_all.jsp?arnumber=17 5794

Chalmers, M. (1995). Design perspectives in visualising complex information. Visual Database Systems (Vol. 3, pp. 103-11). Retrieved from http://pdf.aminer.org/000/591/886/design_of_complex_ information.pdf

Cover, R. (2006). Audience Inter/Active: Interactive Media, Narrative Control \& Reconceiving Audience History. New media \& society, 8(1), 139-158.
Downes, E. J., \& McMillan, S. J. (2000). Defining Interactivity. New Media \& Society, 2(2), $157-179$. doi:10.1177/14614440022225751

Eden, B. (2005). Chapter 1: Information Visualization. Library Technology Reports, 41(1), 7-17.

Elsom-Cook, M. (2001). Principles of Interactive Multimedia. New York: McGrawHill.

Fekete, J. D., Van Wijk, J., Stasko, J., \& North, C. (2008). The value of information visualization. Information Visualization, 1-18.

Gershon, N. D., \& Eick, S. G. (1997). Information Visualization. The Next Frontier. Journal of Intelligent Information Systems, 11(3), 199-204.

Keller, T., \& Tergan, S.-O. (2005). Visualizing Knowledge and Information: An Introduction. In S.-O. Tergan \& T. Keller (Eds.), Knowledge and Information Visualization (Vol. 3426, pp. 1-23). Berlin, Heidelberg: Springer Berlin Heidelberg. Retrieved from http://www.springerlink.com/content/mhnbdl1g9c6w2y cq/

Kiousis, S. (2002). Interactivity: a concept explication. new media \& society, 4(3), 355.

Kirsh, D. (1997). Interactivity and multimedia interfaces. Instructional Science, 25(2), 79-96.

Klanten, R., \& Schardt, J. (2008). Data flow: visualising information in graphic design. Gestalten. Retrieved from http://www.bookforfree.us/PDF/Data-Flow:Visualising-Information-in-Graphic-Design-BY-R.-

Klanten-N.-Bourquin-S.-Ehma-ID533.pdf

Kraidy, U. (2002). Digital media and education: cognitive impact of information visualization. Journal of Educational Media, 27(3), 95-106.

Landow, G. P. (1997). Hypertext 2.0. Johns Hopkins University Press.

Miles, A. (2001). Hypertext structure as the event of connection. Proceedings of the 12th ACM conference on Hypertext and Hypermedia (pp. 61-68).

Purchase, H. C., Andrienko, N., Jankun-Kelly, T. J., \& Ward, M. (2008). Theoretical Foundations of Information Visualization. In A. Kerren, J. T. Stasko, J.-D. Fekete, \& C. North (Eds.), Information Visualization (Vol. 4950, pp. 46-64). Berlin, Heidelberg: Springer Berlin Heidelberg. Retrieved from http://link.springer.com.ezproxy.waikato.ac.nz/chapter/ 10.1007\%2F978-3-540-70956-5_3?LI=true

Timpany, C. (2012). Designing the Printed Book as an Interactive Environment. The International Journal of the Book, 9(1), 11-28.

Tufte, E. R., Goeler, N. H., \& Benson, R. (1990). Envisioning information (Vol. 21). Graphics Press Cheshire, CT.

Vanderschantz, N. (2008). Text Spacing Considerations for Children's On-Screen Reading. Proceedings of World Conference on Educational Multimedia, Hypermedia and Telecommunications 2008 (pp. 16091616). USA: AACE.

Woolman, M. (2002). Digital Information Graphics. New York, NY, USA: Watson-Guptill Publications, Inc. 